Check for updates

Cite this: RSC Adv., 2018, 8, 40581

\title{
Cholesterol-sensing role of phenylalanine in the interaction of human islet amyloid polypeptide with lipid bilayers $\dagger$
}

\begin{abstract}
Ruijie Hao, Yang Li, Liping Guan, Tong Lu, Feihong Meng, Chunyu Wang and Fei Li (D)*
The interactions between hIAPP and the pancreatic $\beta$-cells are associated with $\beta$-cell death in type II diabetes. Cholesterol modulates hIAPP-membrane interaction and hIAPP aggregation. The molecular mechanism underlying this is not well understood. Here we explore the cholesterol-sensing role of F15 in the interactions of hIAPP and hIAPP ${ }_{1-19}$ with various compositions of lipids, including DOPC, DPPC and DOPC/DPPC using NMR, CD, ThT fluorescence and dye leakage assays. We show that both hIAPP and hIAPP $_{1-19}$ are more potent in the disruption to the membranes with cholesterol than they are in the disruption to the membranes without cholesterol. A substitution of F15 by leucine affects the binding and disruption of the peptides to the membranes slightly in the absence of cholesterol, but decreases the activities largely in the presence of cholesterol. F15 also plays a role in accelerating fibrillar assembly of hIAPP, but the function is independent of cholesterol in nature. The promotion of cholesterol to the disruptive potency of hIAPP is more effective in the membrane with raft-like domains than in the membrane with a dispersed distribution of cholesterol. Our results suggest that F15 plays a key role in the cholesterol-sensing binding and disruption of hIAPP to the PC membranes and the distribution of cholesterol in the membranes has an influence on the disruptive activity of hIAPP.
\end{abstract}

Received 2nd September 2018 Accepted 21st November 2018

DOI: $10.1039 / \mathrm{c} 8 \mathrm{ra0} 07310 \mathrm{~d}$

rsc.li/rsc-advances disease. $^{7}$ There is also evidence that Chol is involved in the lipotoxicity of islets underlying the $\beta$-cell dysfunction in T2D. ${ }^{8} \mathrm{~A}$ recent study demonstrates that an elevation in islet Chol promotes IAPP aggregation and islet amyloid formation in mice, worsening $\beta$-cell function and glucose homeostasis. ${ }^{9}$ Studies on model membranes show that the effects of Chol on the interactions between IAPP and membranes and on the amyloidogenesis of IAPP change with the property of membrane. ${ }^{10,11}$

Quite a number of studies have shown that the residues in the N-terminal region of hIAPP play a key role in the interaction of hIAPP with membranes ${ }^{12,13}$ and are involved in the early stages of oligomerization of the peptide. ${ }^{14}$ Therefore, extensive studies have focused on hIAPP ${ }_{1-19}$, a fragment of hIAPP including the 1-19 amino-acid residues. Previous studies have reported that hIAPP ${ }_{1-19}$ is damaging to model membranes composed of pure anionic lipids or mixtures of anionic lipids with zwitterionic lipids, although the peptide is weakly fibrillogenic both in solution and in membrane environment. ${ }^{12,13,15}$ Our recent study demonstrated that the interaction of hIAPP ${ }_{1-19}$ with 1,2-dipalmitoyl-sn-glycero-3-phosphocholine (DPPC) bilayer is promoted by $\mathrm{Chol}$ and the residue phenylalanine at position 15 (F15) plays an important role in the Chol-promoting peptide-membrane interaction. ${ }^{16}$ However, whether the Cholsensing role of F15 establishes for the full length hIAPP peptide is a question to be elucidated. If it establishes indeed, then, how the distribution of Chol in membrane affects the
State Key Laboratory of Supramolecular Structure and Materials, Jilin University, 2699 Qianjin Avenue, Changchun 130012, P. R. China. E-mail: feili@jlu.edu.cn

$\dagger$ Electronic supplementary information (ESI) available. See DOI: $10.1039 / \mathrm{c} 8 \mathrm{ra} 07310 \mathrm{~d}$ 
Chol-sensing role of F15 in the interaction between hIAPP and membrane also needs to be clarified. To find out the answers of these questions, we built up the model membranes using DOPC (1,2-dioleoyl-sn-glycero-3-phosphocholine), DPPC and the 1:2 mixture of DOPC and DPPC and modified the full length hIAPP and hIAPP ${ }_{1-19}$ by F15L substitution in this work.

Both DOPC and DPPC comprise the zwitterionic headgroups, but they are different in the property of the hydrophobic tails. DPPC has two saturated fatty acyl chains, which makes the lipid capable of packing in ordered array in artificial lipid layers and easier to interact with Chol by van der Waals force. ${ }^{17}$ The mixture of DPPC with even relatively small amount of Chol, e.g., $5 \mathrm{~mol} \%$, can result in phase separation, or the coexistence of the liquid-disordered $\left(\mathrm{l}_{\mathrm{d}}\right)$ domain enriched in phospholipids and the liquid-ordered $\left(\mathrm{l}_{\mathrm{o}}\right)$ domain enriched in Chol at a higher temperature or the coexistence of ripple phase $\left(\mathrm{P}_{\beta}\right)$ and $\mathrm{l}_{\mathrm{o}}$ at a lower temperature. ${ }^{18-20}$ On the contrary, DOPC contains two cis-unsaturated fatty acyl chains. Chol increases vertical order and compactness of DOPC bilayer. ${ }^{21-23}$ Phase separation can form only when the molar ratio of Chol/DOPC is increased to $0.75{ }^{24}$ The hybrid membrane built up by DPPC, DOPC and Chol is used frequently as a model membrane in the study of the interaction of amyloid peptide with phospholipid membrane. ${ }^{11,25}$ The ternary mixture is divided into ordered and disordered area, and the size of the ordered region depends on the fraction of $\mathrm{l}_{\mathrm{o}} \cdot{ }^{26}$

By building up different compositions of lipid vesicles and modifying the hIAPP peptides with F15L substitution, we examined the binding and disruption of the hIAPP peptides (hIAPP, hIAPP ${ }_{\mathrm{F} 15 \mathrm{~L}}, \mathrm{hIAPP}_{1-19}$ and hIAPP $_{1-19 / \mathrm{F} 15 \mathrm{~L}}$ ) to the PC membranes in the absence and presence of Chol using various spectroscopic methods. We also monitored the fibrillar assemblies of these peptides in bulk solution and at the PC membranes. Our results demonstrated that not only the Nterminal segment hIAPP $_{1-19}$ but also the full length hIAPP are sensitive to Chol in the interactions with the membranes composed of DOPC, DPPC and DOPC/DPPC, and F15 plays a significant role therein. F15 also plays a significant role in accelerating fibrillar assembly of hIAPP, but the activity is independent of Chol in nature. The disruptive potency of hIAPP is promoted by Chol more efficiently for the membrane with raft-like domains than the membrane with a dispersed distribution of Chol.

\section{Results}

\section{Membrane damage by hIAPP peptides}

The disruptive potencies of the hIAPP peptides to the PC membranes were probed firstly by the leakage assays of the calcein encapsulated LUVs. The addition of either hIAPP or hIAPP $_{\mathrm{F} 15 \mathrm{~L}}$ in the LUV suspended solutions led to an increase in dye release with time. However, the dye leakages induced by hIAPP showed a more rapid increase in the presence of Chol than that in the absence of Chol, whereas the dye leakages induced by hIAPP ${ }_{\mathrm{F} 15 \mathrm{~L}}$ were not considerably different in the absence and presence of $\mathrm{Chol}$ in the entire period of incubation (Fig. 1, panels (A-C)). This indicates that F15 plays a key role in the Chol-sensing disruption of hIAPP to the PC membranes. Furthermore, the results in Fig. 1A-C showed that the differences between the maximal leakages of the Chol-containing and Chol-free LUVs induced by hIAPP are varied with the compositions of lipids from $c a .15-17 \%$ for DPPC and DOPC/DPPC systems to $c a .11 \%$ for DOPC systems. This may be correlated with the distributions of Chol in these membranes.

Compared with the full length hIAPP, hIAPP ${ }_{1-19}$ was much less potent in disrupting the membranes. The leakage percentages reached the plateau in much longer time (several days), even though the $\mathrm{P}: \mathrm{L}$ ratio used in the $\mathrm{hIAPP}_{1-19} / \mathrm{LUV}$ systems $(1: 20)$ was much higher than that used in the hIAPP/ LUV systems $(1: 200)$. Nevertheless, the leaking results of hIAPP $_{1-19} /$ LUV systems (Fig. 1, panels (D-F)) were coincident qualitatively with those of hIAPP/LUV systems. The dye release induced by hIAPP ${ }_{1-19}$ increased with the addition of Chol, while the dye release induced by hIAPP ${ }_{1-19 / \mathrm{F} 15 \mathrm{~L}}$ was less (for DPPC system) or not (for other two lipid systems) affected by the
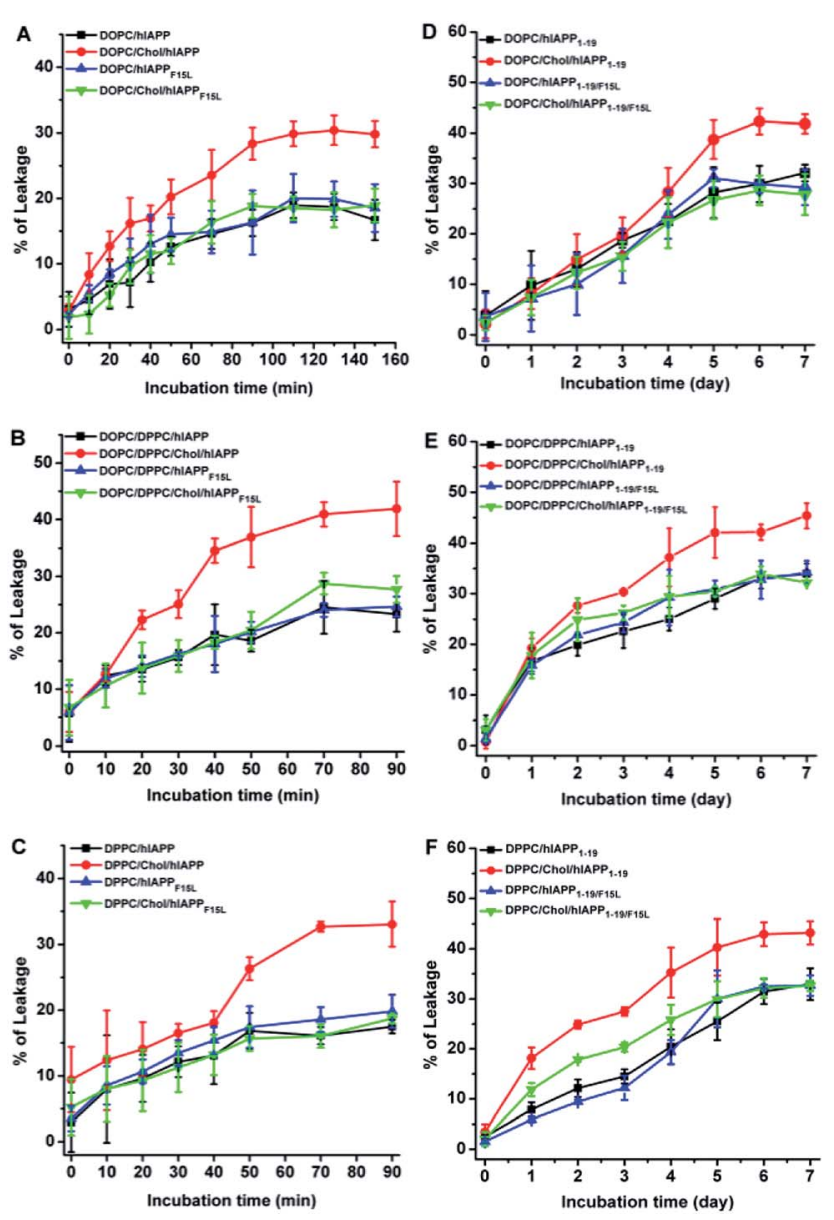

Fig. 1 The leakage results of the LUVs composed of DOPC, DOPC/ 20\% Chol, DOPC/DPPC $1: 2$, DOPC/DPPC/Chol $1: 2: 1$, DPPC and DPPC/20\% Chol in the presence of hIAPP and hIAPP F15L $_{\text {(A-C) }}$ and in the presence of hIAPP $_{1-19}$ and hIAPP $_{1-19 / F 15 L}(D-F)$. The assays were performed in $10 \mathrm{mM}$ Tris- $\mathrm{HCl}$ buffer containing $100 \mathrm{mM} \mathrm{NaCl}$ at $\mathrm{pH}$ 7.4. The concentrations of peptide and total lipids were $15 \mu \mathrm{M}$ and $3 \mathrm{mM}$, respectively, in panels $(\mathrm{A}-\mathrm{C})$, and $75 \mu \mathrm{M}$ and $1.5 \mathrm{mM}$, respectively, in panels (D-F). 

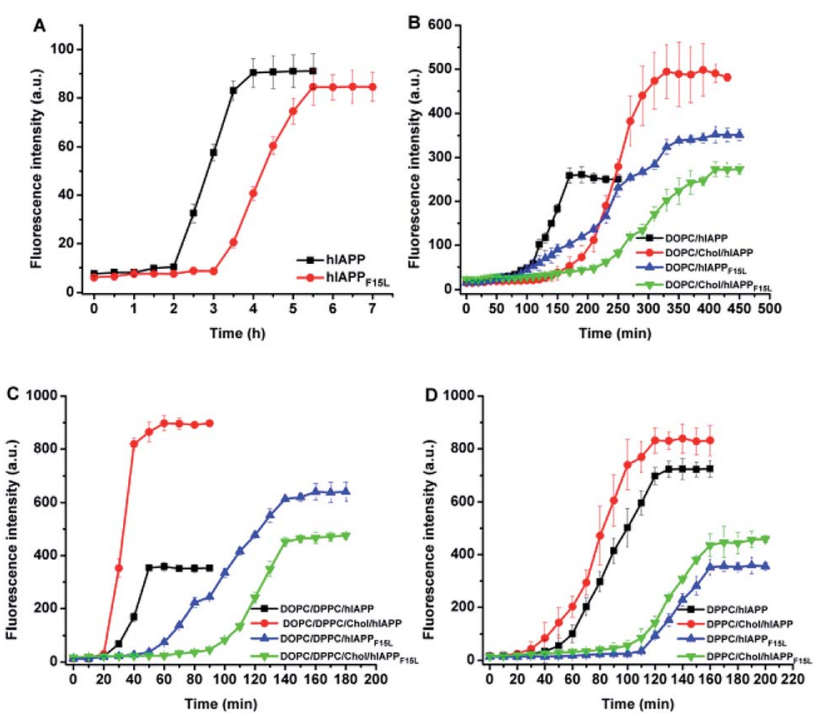

Fig. 2 Time dependences of ThT fluorescence intensity tracking the assemblies of hIAPP and hIAPP $F_{15 L}$ in solution in the absence of LUVs (A) and in the presence of LUVs composed of either DOPC or DOPC/ 20\% Chol (B), DOPC/DPPC $1: 2$ or DOPC/DPPC/Chol $1: 2: 1$ (C), and DPPC or DPPC/20\% Chol (D). The assays were performed in $25 \mathrm{mM}$ phosphate buffer containing $50 \mathrm{mM} \mathrm{NaCl}$ at pH 7.4. The concentrations of peptide and total lipids were $15 \mu \mathrm{M}$ and $3 \mathrm{mM}$, respectively.

presence of Chol. Moreover, an observable difference between the leakages of the Chol-containing and Chol-free LUVs appeared earlier for DPPC and DOPC/DPPC systems (at the 1st and 2nd day of incubation) than DOPC systems (at the 4th day of incubation).

\section{Assemblies of hIAPP peptides at the membranes}

The assemblies of hIAPP and hIAPP ${ }_{\mathrm{F} 15 \mathrm{~L}}$ in solution and at the membranes were monitored by ThT fluorescence assays (Fig. 2). A $25 \mathrm{mM}$ phosphate buffer solution with $50 \mathrm{mM} \mathrm{NaCl}$ was used in the ThT fluorescence assays, replacing a $10 \mathrm{mM}$ Tris- $\mathrm{HCl}$ buffer solution with $100 \mathrm{mM} \mathrm{NaCl}$ used in leaking assays. The changes in buffer solution and salt concentration have no effect on ThT fluorescence results (Fig. S1 $\dagger$ ). Compared with the results of ThT assays of the peptides obtained in the absence of membrane, the intensity of the ThT fluorescence increased dramatically in the presence of the PC membranes. The lag time in the fibrillation kinetics of the peptides was also shortened by the compositions of lipids except for DOPC $/ 20 \%$ Chol which displayed little effect. This indicates that the fibrillar assemblies of both peptides were promoted by the membranes. However, whatever impact the environments exerted, the fibrillar assembly of hIAPP ${ }_{\mathrm{F} 15 \mathrm{~L}}$ was always slower (with longer lag time) than that of hIAPP (with shorter lag time) both in solution and membrane environments. This suggests that the decrease in the fibrillation rate of $\mathrm{F} 15 \mathrm{~L}$ variant relative to that of the wild type peptide arises from the decrease in peptide-peptide interaction in nature, even though the extent of the decrease may vary with environment. In addition, it was noted that the fibrillation of hIAPP is either inhibited by Chol (e.g., at DOPC membrane,
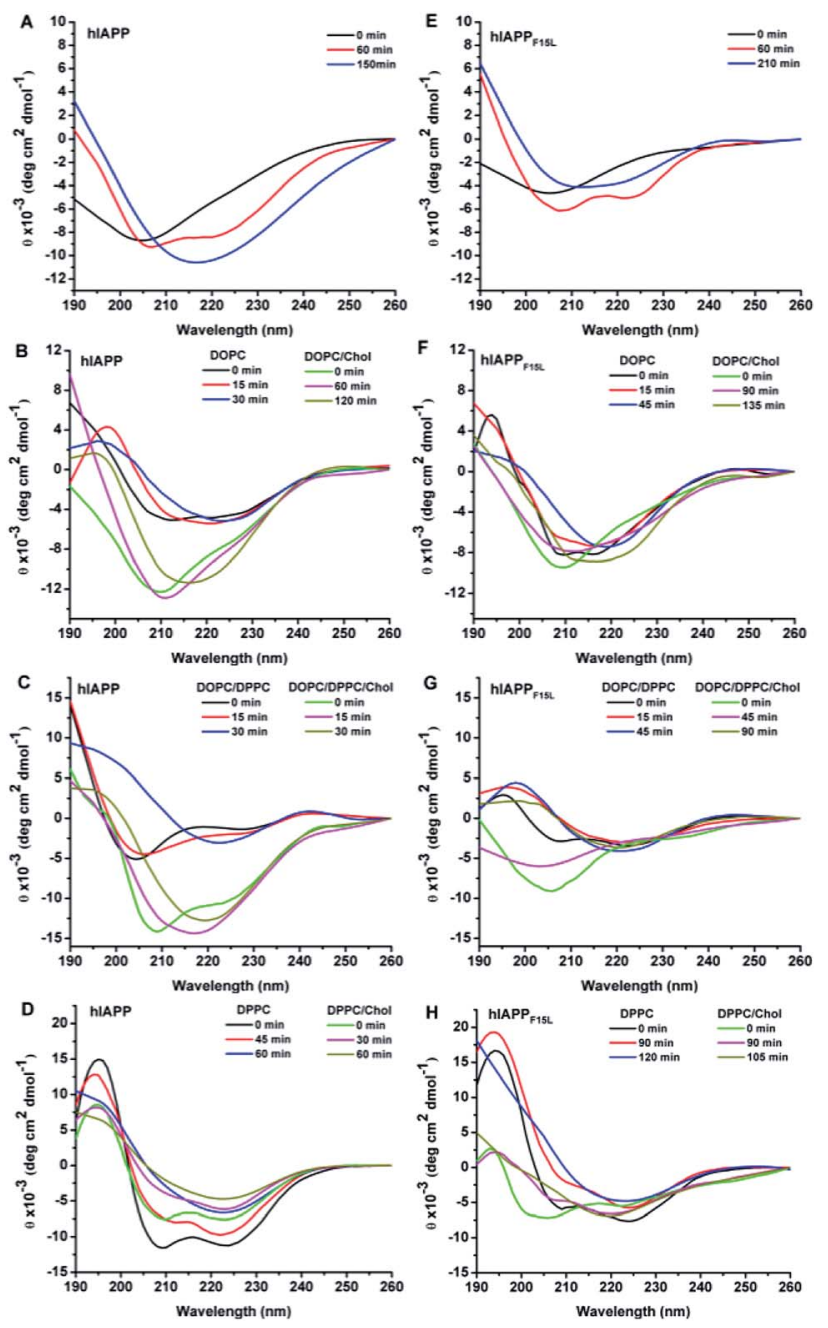

Fig. 3 Representative CD spectra of hIAPP (A-D) and hIAPP ${ }_{F 15 L}(E-H)$ in the absence of LUVs ( $A$ and $E$ ) and in the presence of the LUVs composed of DOPC and DOPC/20\% Chol (B and F), DOPC/DPPC $1: 2$ and DOPC/DPPC/Chol $1: 2: 1$ (C and G), and DPPC and DPPC/20\% Chol $(\mathrm{D}$ and $\mathrm{H})$ at three time points: initial time, the time at which the $\beta$-sheet characteristic absorbance was observed first, and a certain time in between. All the spectra were recorded in $25 \mathrm{mM}$ phosphate buffer at $\mathrm{pH} 7.4$, room temperature, using a concentration of peptide of $15 \mu \mathrm{M}$ and a concentration of total lipids of $3 \mathrm{mM}$.

Fig. 2B $)^{27}$ or promoted by Chol (e.g., at DOPC/DPPC membrane, Fig. 2C), ${ }^{11}$ depending on the composition of membrane, whereas the disruptive potency of hIAPP was always enhanced by Chol in all these membrane systems, as observed in leakage assays. This indicates that the regulation of Chol to the oligomerization/fibrillation of hIAPP is not a direct cause leading to the increase in the disruptive potency of the peptide.

The structural transitions from initial state (random coil or $\alpha$-helix dominant structure) to $\beta$-sheet state were observed in the CD spectra of the two peptides after certain period of incubation either in the absence and in the presence of lipid membranes (Fig. 3, also see Fig. S2, S3, Tables S1 and S2 in ESI $\dagger$ ). Upon mixture with DOPC LUVs, both hIAPP and hIAPP $_{\mathrm{F} 15 \mathrm{~L}}$ showed slower structural transition to $\beta$-sheet in the 

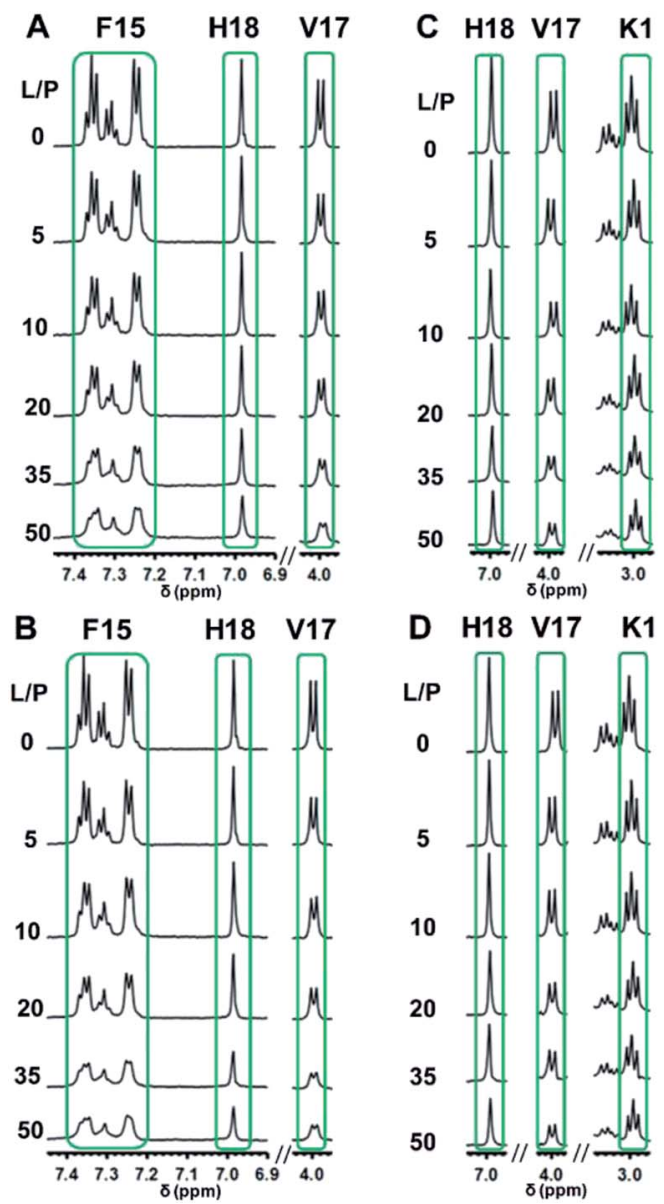

Fig. 4 Representative ${ }^{1} \mathrm{H}-\mathrm{NMR}$ signals of $\mathrm{hIAPP}_{1-19}$ incorporated with DOPC/DPPC $1: 2$ (A) and DOPC/DPPC/Chol $1: 2: 1$ (B) LUVs and those of hIAPP $1-19 /$ F15L incorporated with DOPC/DPPC $1: 2$ (C) and DOPC/DPPC/Chol 1:2:1 (D) LUVs. The spectra were recorded in $25 \mathrm{mM}$ phosphate buffer with $50 \mathrm{mM} \mathrm{NaCl}$ at $\mathrm{pH} 7.4,25^{\circ} \mathrm{C}$.

presence of Chol than in the absence of Chol. Slower structural transition in the presence of Chol than in the absence of Chol was also observed for hIAPP ${ }_{\mathrm{F} 15 \mathrm{~L}}$ mixed with DOPC/DPPC LUVs, while the structural transition was slightly accelerated by Chol for hIAPP incorporated with DOPC/DPPC LUVs. Either hIAPP or hIAPP $_{\mathrm{F} 15 \mathrm{~L}}$, when mixed with DPPC LUVs in the absence and presence of Chol, underwent the structural transition at a similar rate. Moreover, regardless of the effects of Chol, the structural transitions of hIAPP were faster than those of hIAPP $_{\mathrm{F} 15 \mathrm{~L}}$ for all these compositions of lipids. The effects of both Chol and F15L mutation on the rates of $\beta$-sheet formation observed in the CD spectra were basically consistent with those on the rates of fibrillar assemblies observed in ThT fluorescence assays.

It was noted that the differences between the $\mathrm{CD}$ spectra recorded in the absence and presence of Chol are more evident for hIAPP than those recorded for hIAPP ${ }_{\mathrm{F} 15 \mathrm{~L}}$ for all these compositions of lipids (Fig. 3). The secondary structure data showed that the initial structures of the wild type peptide formed in the absence of Chol are largely different from those formed in the presence of Chol, while the initial structures of the F15L variant formed in the absence and presence of Chol
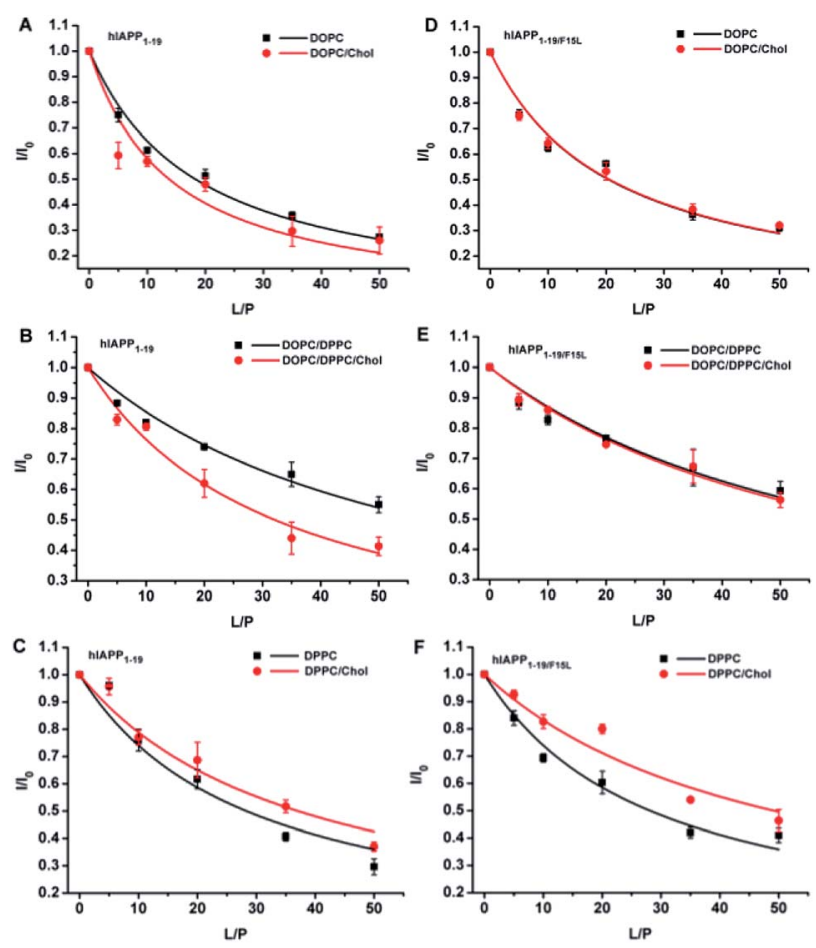

Fig. 5 Dependences of ${ }^{1} \mathrm{H}-\mathrm{NMR}$ intensity on $\mathrm{L}: \mathrm{P}$ ratio for hIAPP ${ }_{1-19}$ and hIAPP 1 19/F15L mixed with various compositions of LUVs (symbols for experimental data and lines for fitting curves). The data were obtained in $25 \mathrm{mM}$ phosphate buffer containing $50 \mathrm{mM} \mathrm{NaCl}$ at pH 7.4, $25^{\circ} \mathrm{C}$.

Table 1 Dissociation constants $\left(K_{\mathrm{d}}\right)$ of $\mathrm{hlAPP}_{1-19}$ and hIAPP $1-19 / \mathrm{F15L}$ binding to LUVs in the absence and presence of Chol obtained by ${ }^{1} \mathrm{H}$ NMR spectra recorded in $25 \mathrm{mM}$ phosphate buffer containing $50 \mathrm{mM}$ $\mathrm{NaCl}$ and $10 \% \mathrm{D}_{2} \mathrm{O}$ at $\mathrm{pH} 7.4,25^{\circ} \mathrm{C}$

\begin{tabular}{lrr}
\hline & \multicolumn{2}{c}{$K_{\mathrm{d}}{ }^{a}(\mathrm{mM})$} \\
\cline { 2 - 3 } Lipid & \multicolumn{1}{c}{ hIAPP $_{1-19}$} & hIAPP $_{1-19 / F 15 L}$ \\
\hline DOPC & $5.34 \pm 0.33$ & $6.00 \pm 0.54$ \\
DOPC/20\% Chol & $3.98 \pm 0.65$ & $6.04 \pm 0.44$ \\
DOPC/DPPC 1 : & $17.41 \pm 1.10$ & $19.84 \pm 1.56$ \\
DOPC/DPPC/Chol 1:2:1 & $9.49 \pm 0.66$ & $19.12 \pm 1.00$ \\
DPPC & $8.33 \pm 1.09$ & $8.28 \pm 0.60$ \\
DPPC/20\% Chol & $10.96 \pm 1.09$ & $14.59 \pm 1.60$
\end{tabular}

${ }^{a}$ The data were obtained from the signals in the regions of 7.20$7.40 \mathrm{ppm}$ (aromatic protons of F15), 6.95-7.03 ppm $\left(\mathrm{H} 18-\mathrm{H}_{\delta}\right)$ and 3.96-4.03 ppm $\left(\mathrm{V} 17-\mathrm{H}_{\alpha}\right)$ for hIAPP ${ }_{1-19}$; 6.95-7.03 ppm $\left(\mathrm{H} 18-\mathrm{H}_{\delta}\right), 3.96-$ $4.03 \mathrm{ppm}\left(\mathrm{V} 17-\mathrm{H}_{\alpha}\right)$ and $2.97-3.02 \mathrm{ppm}\left(\mathrm{K} 1-\mathrm{H}_{\varepsilon}\right)$ for hIAPP ${ }_{1-19 / \mathrm{F} 15 \mathrm{~L}}$, see Fig. 4 and S5-S8 in ESI.

are similar (Table S2 in ESI $\dagger$ ). Considering the results of the leaking assays that the dye releases induced by the wild type peptide are largely different in the absence of Chol from those in presence of Chol, while the dye releases induced by the variant are very similar in the absence and presence of Chol, we infer that the initial assemblies of the peptides formed at various compositions of membrane may play a pivotal role in the membrane disruption. 


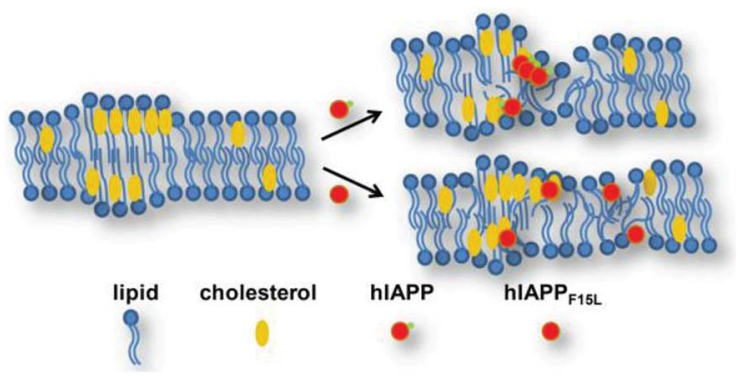

Fig. 6 Schematic mode of mechanism proposed for the interactions between hIAPP/hIAPP ${ }_{F 15 L}$ and the PC membranes in the absence and presence of Chol.

We also monitored the morphologies of the oligomers and fibrils of the two peptides formed in phosphate buffer at $\mathrm{pH} 7.4$ by TEM (Fig. S4 in ESI†). The oligomers of hIAPP and hIAPP ${ }_{\mathrm{F} 15 \mathrm{~L}}$ observed after $30 \mathrm{~min}$ incubation were globular and amorphous, respectively. After $5 \mathrm{~h}$ incubation, the fibrils alone were observed in the TEM image of hIAPP, while the coexistence of fibrils and a large amount of amorphous aggregates was observed in the TEM image of hIAPP $\mathrm{F}_{15 \mathrm{~L}}$.

\section{Binding of hIAPP peptides to the PC membranes}

As hIAPP ${ }_{1-19}$ has a very weak fibrogenic preference and is similar to the full-length peptide in membrane disruption behavior, we determined the binding affinities of hIAPP ${ }_{1-19}$ and hIAPP $_{1-19 / \text { F15L }}$ for the PC membranes using ${ }^{1} \mathrm{H}-\mathrm{NMR}$ measurements. Rapid structural transition and aggregation of the fulllength peptide at these types of membranes, especially at the membrane of DOPC/DPPC $1: 2$, limit the application of the method. The ${ }^{1} \mathrm{H}$-NMR spectra of the peptides incorporated with LUVs were recorded at a series of $\mathrm{L}: \mathrm{P}$ ratios (Fig. 4, $\mathrm{S} 5-\mathrm{S} 8$ in ESI $\dagger$ ) and the intensities of the ${ }^{1} \mathrm{H}$-NMR signals in the selected regions were measured at various $\mathrm{L}: \mathrm{P}$. The dissociation constants $\left(K_{\mathrm{d}}\right)$ of the peptides binding to the membranes with and without Chol were obtained by fitting the data according to eqn (1) (Fig. 5 and Table 1).

The $K_{\mathrm{d}}$ value of hIAPP ${ }_{1-19}$ binding with DOPC/20\% Chol LUVs was smaller than that of the peptide binding with bare DOPC LUVs, while the $K_{\mathrm{d}}$ values of hIAPP ${ }_{1-19 / \mathrm{F} 15 \mathrm{~L}}$ were almost the same for the two compositions of lipids. Similar results were also obtained for the two peptides binding with DOPC/DPPC $1: 2$ and DOPC/DPPC/Chol $1: 2: 1$ LUVs. In contrast, the $K_{\mathrm{d}}$ values of both hIAPP ${ }_{1-19}$ and hIAPP ${ }_{1-19 / \mathrm{F}_{15 \mathrm{~L}}}$ for DPPC LUVs were larger in the presence of $20 \% \mathrm{Chol}$ than those in the absence of Chol. These results indicate that the presence of Chol favors the binding of hIAPP ${ }_{1-19}$ to DOPC-containing LUVs, but disfavors the binding to DPPC LUVs. Nevertheless, the comparison between the $K_{\mathrm{d}}$ values of the two peptides in the same composition of lipids revealed that the binding affinities of hIAPP 1-19 $_{19}$ are similar to those of hIAPP $_{1-19 / \mathrm{F} 15 \mathrm{~L}}$ in the absence of Chol, but higher (with smaller $K_{\mathrm{d}}$ ) than those of hIAPP $_{1-19 / \mathrm{F} 15 \mathrm{~L}}$ in the presence of Chol. This indicates that the substitution of F15L decreases the binding affinity of peptide for the membranes in the presence of Chol, but has little effect on the binding affinity in the absence of Chol. Obviously, F15 plays an important role in the Chol-sensing binding of hIAPP ${ }_{1-19}$ to the membranes. The interaction of hIAPP ${ }_{1-19}$ with Chol may be mediated by F15. A specific interaction of hIAPP ${ }_{1-19}$ with Chol in DPPC could partially balance the disadvantage in binding. This could explain why the presence of Chol in DPPC LUVs induces a smaller increase in $K_{\mathrm{d}}$ of hIAPP $_{1-19}$ than that of hIAPP ${ }_{1-19 / F 15 \mathrm{~L}}$ relative to the $K_{\mathrm{d}}$ values obtained in the absence of Chol.

With the increase in $\mathrm{L}: \mathrm{P}$, only the decrease in intensity was observed, while the change in chemical shift was not observed for all signals in ${ }^{1} \mathrm{H}$-NMR spectra. This indicates that the binding of the peptides with these PC membranes is very weak. This may be attributed to the lack of anionic component needed for a stronger electrostatic interaction between peptide and membrane. The $K_{\mathrm{d}}$ data obtained by $1: 1$ binding mode are in the $\mathrm{mM}$ order of magnitude, also showing a very weak binding. This means that the estimation of $K_{\mathrm{d}}$ by the simple binding mode is reasonable for the peptide-lipid systems used in this study, even though the method, to a large extent, is an approximation for the complex peptide-membrane systems.

\section{Discussion}

The results above demonstrate that F15 is significant for hIAPP in the fibril formation, membrane binding, and membrane disruption. It is interesting that the promotion role of F15 to the fibril formation of hIAPP is effective at the membranes both with and without Chol, while the promotion roles of F15 to the membrane binding and membrane disruption are effective only at the membranes with Chol. This suggests that the promotion role of F15 in fibrillar assembly is independent of Chol in nature, while the promotion roles of F15 in membrane binding and membrane damage may be associated with specific interaction of the peptide with Chol.

It is noted that the hIAPP-induced dye release occurs earlier than the fibril formation or even the structural transition to $\beta$ sheet for all these peptide/LUV systems. This confirms that the oligomers instead of fibrils are the disruptive species of the peptides. In addition, the disruptive potency of hIAPP is always enhanced by Chol for all the lipid systems, while the fibrillation of the peptide is either accelerated or restricted by Chol, depending on the composition of lipids. This implies that the oligomeric species formed off the fibrillation pathway may be responsible for the disruptive activity of hIAPP at these PC membranes. The interfering of F15L mutation to the peptide oligomerization/fibrillation could not be a direct cause leading to the decrease in the Chol-sensing activity in membrane disruption. Otherwise, the difference between the leaking results of the two peptides should also be observed for the membranes without Chol. This is not true obviously.

The model membranes used in this study contain different fractions of saturated and unsaturated components, which leads to different distributions of $\mathrm{Chol}$ in the membranes. Chol is prone to clustering to form raft-like domains in the lipid membranes with saturated acyl chains, e.g., DPPC $/ 20 \%$ Chol and DOPC/DPPC/Chol 1:2:1 membrane. Therefore, a phase separation of the micro-area enriched in Chol from that enriched in lipids occurs. A curvature strain resulting from 
different membrane thicknesses between the Chol-rich and Chol-poor domains may exist, which renders the domain boundaries more vulnerable to membrane disrupting agents. ${ }^{11,28}$ In contrast, Chol distributes in the membrane with unsaturated acyl chains, e.g., DOPC membrane, more dispersedly and no phase separation occurs at the concentration used in this study. The leaking results of both hIAPP and hIAPP $_{1-19}$ reveal that DOPC/DPPC and DPPC LUVs are more effectively promoted by Chol in dye release than DOPC LUVs. This suggests that the disruptive potency of hIAPP/hIAPP ${ }_{1-19}$ is promoted by Chol more efficiently in the membranes with raftlike domains than in the membrane without the domains. The recognition of hIAPP/hIAPP ${ }_{1-19}$ to Chol mediated by F15 may play a significant role therein. The recognition of hIAPP/hIAPP ${ }_{19}$ to Chol in the raft-like domains could facilitate the aggregation of the peptides at the Chol-rich area or the boundary region of the domains. The interaction of hIAPP/hIAPP ${ }_{1-19}$ with aggregated Chol may also facilitate the formation of damaging oligomers more efficiently than the interaction with dispersedly distributed Chol in the membrane (Fig. 6).

The $K_{\mathrm{d}}$ data show that hIAPP ${ }_{1-19}$ binds to the membranes with a higher affinity than hIAPP ${ }_{1-19 / F 15 L}$ does in the presence of Chol. A higher level of dye release induced by hIAPP ${ }_{1-19}$ than by hIAPP $_{1-19 / F 15 L}$ for the membranes with Chol was also observed for these compositions of lipids. However, a stronger binding of hIAPP $_{1-19}$ to DPPC LUVs (smaller $K_{\mathrm{d}}$ ) than to DPPC/20\% Chol (a larger $K_{\mathrm{d}}$ ) is related to a less efficient disruption of the former than the latter by the peptide. This indicates that a positive correlation of disruptive efficiency with binding affinity only establishes for the peptide (hIAPP $_{1-19}$ or hIAPP) at the membrane with the same composition of lipids, but is not necessarily true when the comparison is done between the membranes with different compositions of lipids.

\section{Conclusions}

The presence of Chol in the membranes composed of DOPC, DPPC and DOPC/DPPC enhances the susceptibility of the membranes to hIAPP/hIAPP ${ }_{1-19}$. The unfavorable effect of Chol on the stability of the membranes is mainly attributed to a prone interaction of the hIAPP peptides with Chol. The existence of raft-like domains enlargers the susceptibility of the membranes to the peptides, likely by increasing curvature strain of the membranes or promoting the formation of more damaging oligomers of the peptides. The aromatic residue phenylalanine at position 15 plays a pivotal role in the specific interaction of the peptides with Chol. Without the aromatic ring, hIAPP loses the Chol-sensing potency in membrane disruption partially or even totally whether the raft-like domains form or not.

\section{Experimental}

\section{Materials}

The peptides KCNTATCATQRLANFLVHSSNNFGAILSSTNVGSNTY (hIAPP), KCNTATCATQRLANFLVHS $\left(\right.$ hIAPP $\left._{1-19}\right)$, and the F15L variants of the peptides hIAPP $_{\mathrm{F} 15 \mathrm{~L}}$ and hIAPP ${ }_{1-19 / F 15 L}$ ) were synthesized by Shanghai Sci. Pep. Biol. Technol. Co., Ltd. (Shanghai, China). The cysteines at positions 2 and 7 were oxidized to form a disulfide bond and the $\mathrm{C}$ termini were amidated in the peptides. The purity of the peptides was assessed by high performance liquid chromatography and mass spectroscopy, and the peptide power with a purity greater than 95\% was obtained. Phospholipids DOPC and DPPC were obtained from Avanti Polar Lipid, Inc. (Alabaster, AL). Other chemical agents were purchased from Sigma-Aldrich (St. Louis, MO).

\section{Pre-treatment of peptide}

The synthesized peptide was dissolved in 1,1,1,3,3,3-hexafluoro2-isopropanol (HFIP) solution at a concentration of $1 \mathrm{mg} \mathrm{mL}^{-1}$ and sonicated in a water bath for 1 hour at a temperature of $30{ }^{\circ} \mathrm{C}$ to disrupt the pre-aggregated assembly. After sonication, the peptide was lyophilized overnight.

\section{Preparation of large unilamellar lipid vesicles (LUVs)}

Certain quantities of phospholipids (DOPC, DPPC and Chol) were dissolved in chloroform/methanol $(2: 1 \mathrm{v} / \mathrm{v})$ co-solvent separately and mixed with desired ratios. The mixtures were evaporated by a stream of nitrogen, and then kept under vacuum overnight. The dried lipid film was hydrated either with phosphate buffer or with $1 \mathrm{~mL}$ Tris-HCl buffer containing $70 \mathrm{mM}$ calcein $(\mathrm{NaOH}$ of $5 \mathrm{M}$ was then added dropwise until the solution turned clear). After $1 \mathrm{~h}$ incubation at $\sim 30{ }^{\circ} \mathrm{C}$ (for DOPC and DOPC/Chol) or at $\sim 55^{\circ} \mathrm{C}$ (for DPPC-containing lipids), the solution was freeze-thawed 15 times and extruded 10 cycles through polycarbonate filter of $0.1 \mu \mathrm{m}$. The calcein encapsulated LUVs were further dialyzed through a membrane with a cut-off of $1000 \mathrm{Da}$ in buffer for three days to eliminate the nonencapsulated calcein.

\section{NMR spectroscopy}

${ }^{1} \mathrm{H}-\mathrm{NMR}$ spectra were performed on a Bruker Avance $600 \mathrm{spec}-$ trometer (Bruker Bio-Spin, Fällanden, Switzerland) at $25{ }^{\circ} \mathrm{C}$. In the ${ }^{1} \mathrm{H}$-NMR experiments, the LUVs suspended in $25 \mathrm{mM}$ phosphate buffer containing $50 \mathrm{mM} \mathrm{NaCl}$ and $10 \% \mathrm{D}_{2} \mathrm{O}$ at $\mathrm{pH}$ 7.4 were used. The peptide concentration was fixed to $300 \mu \mathrm{M}$ (for DOPC and DOPC/DPPC LUV systems) or $60 \mu \mathrm{M}$ (for DPPC LUV systems), changing lipid concentrations to obtain the molar ratios of lipid-to-peptide (L : P) from 0 to 50. The spectra were collected with scans of 256 (for DOPC and DOPC/DPPC LUV systems) or 512 (for DPPC LUV systems) and a relaxation delay of 3 s. DSS (2,2-dimethyl-2-silapentane-5-sulfonate- $\mathrm{d}_{6}$ ) was used as an internal standard. The ${ }^{1} \mathrm{H}-\mathrm{NMR}$ intensities of peptide were measured at various $\mathrm{L}: \mathrm{P}$ and the dissociation constants $\left(K_{\mathrm{d}}\right)$ of peptide binding to LUVs were estimated by eqn (1) derived from a simple bimolecular binding equilibrium: ${ }^{10,29}$

$$
I=I_{0}\left(1-\frac{\left(\frac{K_{\mathrm{d}}}{P_{0}}+x+1\right)-\sqrt{\left(\frac{K_{\mathrm{d}}}{P_{0}}+x+1\right)^{2}-4 x}}{2}\right)
$$


where $I$ is the observed intensity of a selected ${ }^{1} \mathrm{H}$ resonance signal and $I_{0}$ is the maximum intensity of this ${ }^{1} \mathrm{H}$ signal. $P_{0}$ refers to the concentration of peptide, and $x$ represents the molar ratio of lipid-to-peptide.

\section{Circular dichroism (CD) spectroscopy}

CD measurements were carried out using a PMS-450 spectropolarimeter (Biologic, France) at room temperature using a quartz cuvette with $0.5 \mathrm{~mm}$ path length under a constant flow of $\mathrm{N}_{2}$. Peptide was dissolved in $25 \mathrm{mM}$ phosphate buffer at $\mathrm{pH}$ 7.4 and the CD spectra were recorded in the absence and presence of LUVs at room temperature. The concentration of peptide was $15 \mu \mathrm{M}$ and the total concentration of lipids was $3 \mathrm{mM}$. The $\mathrm{CD}$ experiments were performed in a scan range from $190 \mathrm{~nm}$ to $260 \mathrm{~nm}$ at a scan rate of $1 \mathrm{~nm}$ in an interval of $10 \mathrm{~s}$. Three independent experiments were collected and averaged. The blank spectra were subtracted. The contents of secondary structure components of peptide were calculated by CDPro software package using program CONTIN/LL.

\section{Membrane leakage assays}

The membrane leakage assays were performed on a fluorescence spectrophotometer RF-5301 PC (Shimadzu, Japan) at room temperature using an excitation wavelength of $495 \mathrm{~nm}$ and an emission wavelength from $530 \mathrm{~nm}$ to $650 \mathrm{~nm}$. After peptide was mixed with LUVs in $10 \mathrm{mM}$ Tris-HCl buffer con-

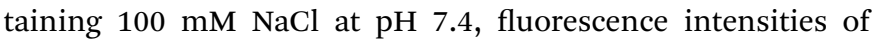
calcein released from LUVs were measured at different incubation time and analysed using eqn (2): $:^{30-32}$

$$
\% \text { Dye leakage }=\frac{I-I_{0}}{I_{100}-I_{0}} \times 100
$$

where $I$ and $I_{0}$ represent the observed fluorescence intensities of the calcein-LUVs in the presence and absence of peptide, respectively. $I_{100}$ was the $100 \%$ leakage obtained by the calceinLUVs with $0.2 \%$ Triton X-100. The concentrations of peptide and total lipids were $75 \mu \mathrm{M}$ and $1.5 \mathrm{mM}$, respectively, for the leakage assays of hIAPP ${ }_{1-19}$, and $15 \mu \mathrm{M}$ and $3 \mathrm{mM}$, respectively, for the leakage assays of hIAPP.

\section{Thioflavin-T fluorescence assays}

The Thioflavin-T fluorescence assays were carried out on a fluorescence spectrophotometer RF-5301 PC (Shimadzu, Japan) at room temperature using an excitation wavelength of $440 \mathrm{~nm}$ and an emission wavelength from $450 \mathrm{~nm}$ to $600 \mathrm{~nm}$. A slit of $3 \mathrm{~nm}$ was used in the scanning process. Peptide was dissolved in $25 \mathrm{mM}$ phosphate buffer ( $50 \mathrm{mM} \mathrm{NaCl}, \mathrm{pH} 7.4)$ containing $20 \mu \mathrm{M}$ ThT and the fluorescence assays were performed in the absence and presence of LUVs at room temperature. The concentration of peptide was $15 \mu \mathrm{M}$ and the total concentration of lipids was $3 \mathrm{mM}$.

\section{Transmission electron microscopy (TEM)}

A peptide solution of $15 \mu \mathrm{M}$ was diluted to $3 \mu \mathrm{M}$ after incubation for $30 \mathrm{~min}$ or $5 \mathrm{~h}$. A $5 \mu \mathrm{L}$ of the solution was applied on a 300- mesh Formvar/carbon coated copper grid. After waiting for $10 \mathrm{~min}$, the sample was washed twice with $10 \mathrm{~mL}$ phosphate buffer. The sample was air-dried overnight. TEM images were observed under a transmission electron microscope (JEM2100F, JEOL Co., Ltd, Japan) operating at an accelerating voltage of $200 \mathrm{kV}$.

\section{Conflicts of interest}

There are no conflicts of interest to declare.

\section{Acknowledgements}

This work was supported by the National Natural Science Foundation of China (grant number 21673099).

\section{References}

1 A. Abedini and A. M. Schmidt, FEBS Lett., 2013, 587, 11191127.

2 F. M. Ashcroft and P. Rosman, Cell, 2012, 148, 1160-1171.

3 J. Fantini, N. Garmy, R. Mahfoud and N. Yahi, Expert Rev. Mol. Med., 2002, 4, 1-22.

4 J. P Liu, Y. Tang, S. Zhou, B. H. Toh, C. Mclean and H. Li, Mol. Cell. Neurosci., 2010, 43, 33-42.

5 J. Fantini and N. Yahi, Expert Rev. Mol. Med., 2010, 12, e27.

6 I. J. Martins, T. Berger, M. J. Sharman, G. Verdile, S. J. Fuller and R. N. Martins, J. Neurochem., 2009, 111, 1275-1308.

7 L. Mascitelli, F. Pezzetta and M. R. Goldstein, Neurology, 2008, 70, 1972-1979.

8 L. R. Brunham, J. K. Kruit, M. R. Hayden and C. B. Verchere, Curr. Diabetes Rep., 2010, 10, 55-60.

9 N. Wijesekara, A. Kaur, C. Westwell-Roper, D. Nackiewicz, G. Soukhatcheva, M. R. Hayden and C. B. Verchere, Diabetologia, 2016, 59, 1242-1246.

10 L. Caillon, L. Duma, O. Lequin and L. Khemtemourian, Mol. Membr. Biol., 2014, 31, 239-249.

11 M. F. Sciacca, F. Lolicato, G. Di Mauro, D. Milardi, L. D'Urso, C. Satriano, A. Ramamoorthy and C. La Rosa, Biophys. J., 2016, 111, 140-151.

12 L. Khemtémourian, M. F. M. Engel, R. M. J. Liskamp, J. W. M. Höppener and J. A. Killian, Biochim. Biophys. Acta, 2010, 1798, 1805-1811.

13 J. R. Brender, E. L. Lee, M. A. Cavitt, A. Gafni, D. G. Steel and A. Ramamoorthy, J. Am. Chem. Soc., 2008, 130, 6424-6429.

14 Y. Mazor, S. Gilead, I. Benhar and E. Gazit, J. Mol. Biol., 2002, 322, 1013-1024.

15 D. L. Heyl, J. M. Osborne, S. Pamarthy, S. Samisetti, A. W. Gray, A. Jayaprakash, S. Konda, D. J. Brown, S. R. Miller, R. Eizadkhah and M. C. Milletti, Int. J. Pept. Res. Ther., 2010, 16, 43-54.

16 Y. Li, L. Guan, T. Lu, H. Li, Z. Li and F. Li, RSC Adv., 2016, 6, 96837-96846.

17 J. Fantini and F. J. Barrantes, Biochim. Biophys. Acta, 2009, 1788, 2345-2361.

18 T. P. W. Mcmullen, R. N. A. H. Lewis and R. N. Mcelhaney, Biochemistry, 1993, 32, 516-522. 
19 T. P. W. Mcmullen and R. N. Mcelhaney, Biochim. Biophys. Acta, 1995, 1234, 90-98.

20 M. Eeman and M. Deleu, Biotechnol., Agron., Soc. Environ., 2010, 14, 719-736.

21 E. Drolle, N. Kučerka, M. I. Hoopes, Y. Choi, J. Katsaras, M. Karttunen and Z. Leonenko, Biochim. Biophys. Acta, Biomembr., 2013, 1828, 2247-2254.

22 M. Alwarawrah, J. Dai and J. Huang, J. Phys. Chem. B, 2010, 114, 7516-7523.

23 M. Jurak, J. Phys. Chem. B, 2013, 117, 3496-3502.

24 M. Jurak and E. Chibowski, RSC Adv., 2015, 5, 66628-66635.

25 K. Weise, D. Radovan, A. Gohlke, N. Opitz and R. Winter, ChemBioChem, 2010, 11, 1280-1290.

26 R. F. M. de Almeida, L. M. S. Loura, A. Fedorov and M. Prieto, J. Mol. Biol., 2005, 346, 1109-1120.
27 L. Caillon, O. Lequin and L. Khemtemourian, Biochim. Biophys. Acta, Biomembr., 2013, 1828, 2091-2098.

28 P. E. S Smith, J. R. Brender and A. Ramamoorthy, J. Am. Chem. Soc., 2009, 131, 4470-4478.

29 C. M. Pfefferkorn and J. C. Lee, J. Phys. Chem. B, 2010, 114, 4615-4622.

30 D. Milardi, M. F. M. Sciacca, M. Pappalardo, D. M. Grasso and C. L. Rosa, Eur. Biophys. J., 2011, 40, 1-12.

31 M. F. M. Engel, L. Khemtémourian, C. C. Kleijer, H. J. D. Meeldijk, J. Jacobs, A. J. Verkleij, B. de Kruijff, J. A. Killian and J. W. M. Höppener, Proc. Natl. Acad. Sci. U. S. A., 2008, 105, 6033-6038.

32 C. A. D. Carufel, N. Quittot, P. T. Nguyen and S. Bourgault, Angew. Chem., Int. Ed., 2015, 54, 14383-14387. 